\title{
A note on the uncertainty in tsunami shape for estimation of its run-up heights
}

\author{
Ira Didenkulova · Oleg Didenkulov · Efim Pelinovsky
}

Received: 23 November 2014 / Accepted: 18 February 2015 / Published online: 13 March 2015

(C) Springer International Publishing AG 2015

\begin{abstract}
In practice, when tsunami approaches the coast and the time for decision making and issuing warning alert is limited, design formulas for fast estimation of tsunami runup characteristics are applied. The most famous and the most used among them assume that incoming wave has a solitonic shape. However, the exact shape of the incoming wave is usually unknown. This is why it is important to know the error caused by the wave shape uncertainty. In this paper, we discuss how the uncertainty of the incoming wave shape influences its run-up characteristics in different bays. Two typical beach geometries: plane beach and U-shaped bay are considered.
\end{abstract}

Keywords Tsunami $\cdot$ Run-up · Uncertainty in wave shape $\cdot$ Parameterization $\cdot$ Nonlinear shallow water theory

I. Didenkulova $(\varangle) \cdot$ O. Didenkulov $\cdot$ E. Pelinovsky

Nizhny Novgorod State Technical University n.a. R.E. Alekseev, Minin Street 24, 603950 Nizhny Novgorod, Russia

e-mail: ira@cs.ioc.ee

I. Didenkulova

Institute of Cybernetics at Tallinn University of Technology,

Akadeemia tee 21, 12618 Tallinn, Estonia

E. Pelinovsky

Institute of Applied Physics, Ulyanov Street 46,

603950 Nizhny Novgorod, Russia

E. Pelinovsky

National Research University, Higher School of Economics,

Bolshaya Pecherskaya 25, 603950 Nizhny Novgorod, Russia

E. Pelinovsky

Special Research Bureau for Automatization of Marine Research, Gorky 25, 693013 Uzhno-Sakhalinsk, Russia

\section{Introduction}

Tsunami science progressed very much after the catastrophic 2004 Indian Ocean tsunami. Existing tsunami early warning systems (TEWS) have been improved and new ones, e.g., German-Indonesian tsunami early warning system (Lauterjung et al. 2010), have been developed. Although the propagation of tsunami in the open ocean is no longer a problem, the reliable estimate of tsunami run-up characteristics on a beach still remains a challenge. To save calculation time, instead of running detailed simulation of wave run-up in the real coastal topography, simplified formulas for wave run-up based on parameters of incoming wave are applied. These formulas based on known analytical solutions for solitary waves, e.g., run-up of a soliton (Synolakis 1987), or represent a set of pre-calculated amplification factors for different incoming wave scenarios (Løvholt et al. 2012). However, in all these cases the detailed shape of the incoming tsunami wave remains unknown and its parameters (wave height and wavelength) are either pre-computed using different hydrodynamic models or estimated from the first wave measurements, such as DART buoys. As having reliable method for calculation of tsunami run-up characteristics is critical for estimation of tsunami inundation zone and impact on port and coastal structures, it is important to introduce the confidence interval related to the uncertainty in the wave shape. This is done in the given paper for a set of solitary pulses propagating in two different bottom geometries: the classical plane beach and a U-shaped bay.

The plane beach is the most studied bottom configuration in tsunami run-up research. After the pioneer paper by Carrier and Greenspan (Carrier and Greenspan 1958) who obtained first rigorous mathematical results for long wave run-up on a plane beach, a number of analytical solutions for various shapes of incident waves have been found (Spielvo- 
gel 1975; Pedersen and Gjevik 1983; Synolakis 1987; Pelinovsky and Mazova 1992; Tadepalli and Synolakis 1994; Brocchini and Gentile 2001; Carrier et al. 2003; Kânoğlu 2004; Tinti and Tonini 2005; Kânoğlu and Synolakis 2006; Antuono and Brocchini 2007, 2008, 2010; Didenkulova et al. 2007a, b; Madsen and Fuhrman 2008; Didenkulova 2009). Even though the geometry sketch is rather simple, the problem is still far from being solved and more research on wave run-up on a plane beach is still needed (Pedersen et al. 2013).

However, estimates of run-up characteristics calculated for a plane beach cannot be applied to long and narrow Ushaped bays as it was observed during Samoa 2009, when the observed run-up heights significantly exceeded the one estimated by the formulas for a plane beach (Okal et al. 2010; Didenkulova 2013). Later, it has been demonstrated that estimates of run-up characteristics performed using Ushaped bay approach are in a good agreement with observations of tsunami run-up height in Pago-Pago, American Samoa (Didenkulova 2013). All this suggests that U-shaped bays should be considered independently. U-shaped bays have been considered in studies by (Aranguiz and Shibayama 2013; Didenkulova and Pelinovsky 2011a, b; Rybkin et al. 2014; Vasskog et al. 2013).

In this paper we estimate an error related to the uncertainty in tsunami shape, by considering a set of solitary waves climbing a plane beach or the coast of U-shaped bay. The results for the plane beach have been previously obtained by (Didenkulova et al. 2007a, 2008a) and we reproduce them here to compare with the novel results for U-shaped bay. To provide a feasible comparison, we reproduce main formulas and equations for solitary wave run-up on a plane beach leaving the details aside. The calculations are performed in the framework of nonlinear shallow water theory. The paper is organized as follows. In Sect. 2, we give an insight to the problem of wave run-up on a plane beach and discuss the differences in run-up characteristics caused by uncertainties in the incoming wave shape (the error related to the difference in wave shapes). Similarly, in Sect. 3, wave run-up in $\mathrm{U}$-shaped bay is described and the corresponding errors due to run-up of waves of different shapes are calculated. Section 4 is devoted to the effects of wave asymmetry and steepness of the incoming wave front. Main results are summarized in Sect. 5 .

\section{Uncertainty in wave run-up characteristics on a plane beach}

In this section, we calculate run-up characteristics of bellshaped waves and parameterize the corresponding formulas for long waves of "unknown" shape by reproducing main results of (Didenkulova et al. 2007a, 2008a).

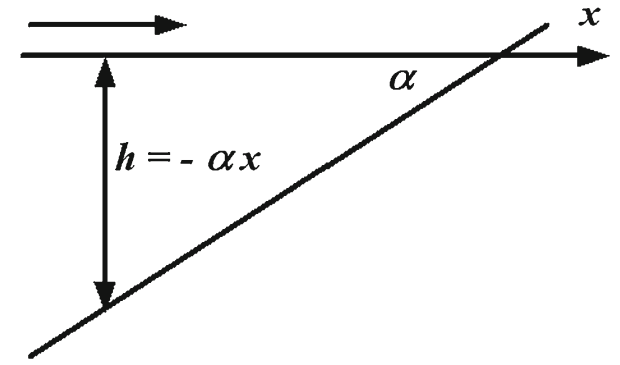

Fig. 1 Geometry sketch for wave run-up on a plane beach

Shallow water equations for a plane beach have the form:

$\frac{\partial \eta}{\partial t}+\frac{\partial}{\partial x}[(-\alpha x+\eta) u]=0, \quad \frac{\partial u}{\partial t}+u \frac{\partial u}{\partial x}+g \frac{\partial \eta}{\partial x}=0$,

where $\eta$ is the level oscillations, $u$ is averaged over water depth flow velocity, $\alpha$ is the slope angle, $x$-coordinate directed onshore, $t$ is time and $g$ is the gravitational acceleration. The geometry sketch is shown in Fig. 1.

System of Eq. (1) can be solved using the hodograph transformation. This procedure was first described by Carrier and Greenspan (1958), and later reproduced in different papers cited in Introduction. According to this method, all original variables $(\eta, u, x, t)$ can be expressed through the "nonlinear" wave function $\Phi(\sigma, \lambda)$ and new variables $\lambda$ and $\sigma$ :

$\eta=\frac{1}{2 g}\left(\frac{\partial \Phi}{\partial \lambda}-u^{2}\right), \quad u=\frac{1}{\sigma} \frac{\partial \Phi}{\partial \sigma}$,
$t=\frac{1}{\alpha g}\left(\lambda-\frac{1}{\sigma} \frac{\partial \Phi}{\partial \sigma}\right), \quad x=\frac{1}{2 \alpha g}\left(\frac{\partial \Phi}{\partial \lambda}-u^{2}-\frac{\sigma^{2}}{2}\right)$,

and the wave function, $\Phi(\lambda, \sigma)$ satisfies to the cylindrical linear wave equation

$\frac{\partial^{2} \Phi}{\partial \lambda^{2}}-\frac{\partial^{2} \Phi}{\partial \sigma^{2}}-\frac{1}{\sigma} \frac{\partial \Phi}{\partial \sigma}=0$

Variables $\lambda$ and $\sigma$ have meaning of generalized coordinates. As $\sigma=2 \sqrt{g H} \geq 0$, where $H=\eta-\alpha x$ is the total water depth, the point $\sigma=0$ corresponds to the instantaneous position of the moving shoreline. And, therefore, the function

$r(t)=\eta(t, \sigma=0)$

describes the desired oscillations of the vertical displacement of the moving shoreline for any kind of initial condition. Following kinematical consideration, the velocity of the shoreline motion, $u_{r}$, can be found as a time derivative of Eq. (5):

$u_{r}(t)=\frac{1}{\alpha} \frac{\mathrm{d} r(t)}{\mathrm{d} t}$ 


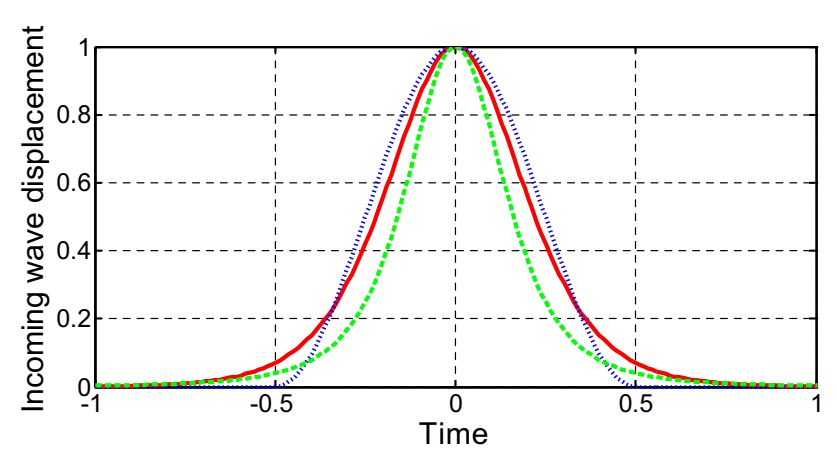

Fig. 2 Examples of bell-shaped waves described by Eq. (7) for $n=2$ : sine-like wave (blue dotted), soliton (red solid) and Lorentz-like (green dashed line) (color figure online)

To study the impact of uncertainty of the incident wave shape, we consider 58 different waves (sine-like, soliton-like and Lorentz-like)

$$
\begin{aligned}
& \eta_{0}(t)=A \cos ^{n}(\pi t / T), \quad \eta_{0}(t)=A \sec h^{n}(t / T), \\
& \eta_{0}(t)=\frac{A}{\left[1+(t / T)^{2}\right]^{n}},
\end{aligned}
$$

where $A$ and $T$ are amplitude and period of the incoming wave, $n=1,2, \ldots, 20$ for waves of all shapes except the sine-like, for which $n=3,4, \ldots, 20$. All waves described by Eq. (7) have a bell-like shape with some small variations in its shape (see Fig. 2). The influence of these variations is studied here.

We are interested only in extreme characteristics of wave run-up, such as maximal run-up and run-down heights, and run-up and run-down velocities. To estimate the difference in run-up heights caused by variations in the incoming wave shape, the following parameterization has been suggested (Didenkulova et al. 2007a, 2008a):

$R_{\text {up }}=\mu_{R+} A \sqrt{L / \lambda_{s}}, \quad R_{\text {down }}=\mu_{R-} A \sqrt{L / \lambda_{s}}$,

where $R_{\text {up }}$ and $R_{\text {down }}$ are maximal run-up and run-down heights, respectively, $L$ is the travel distance to shore, $\lambda_{s}$ is the "significant" wavelength defined at the $2 / 3$ level of the maximum wave height, and $\mu_{R+}$ and $\mu_{R-}$ are the wave shape coefficients. Numerical coefficients $\mu$ reflect differences in shape of all considered waves [Eq. (7)] and allow their comparison. For example, using relation between wave amplitude and its wave length for a soliton, and substituting the corresponding coefficients $\mu$ in Eq. (8), one can obtain famous expression for solitary wave run-up (Synolakis 1987). Also, coefficients $\mu$ for Lorentz pulse $(n=1)$ coincide with those in the formula obtained by Pelinovsky and Mazova (1992).

Similarly to Eq. (8), we can introduce $\mu_{U+}$ and $\mu_{U-}$, wave shape parameters in the expressions for extreme shoreline velocities:

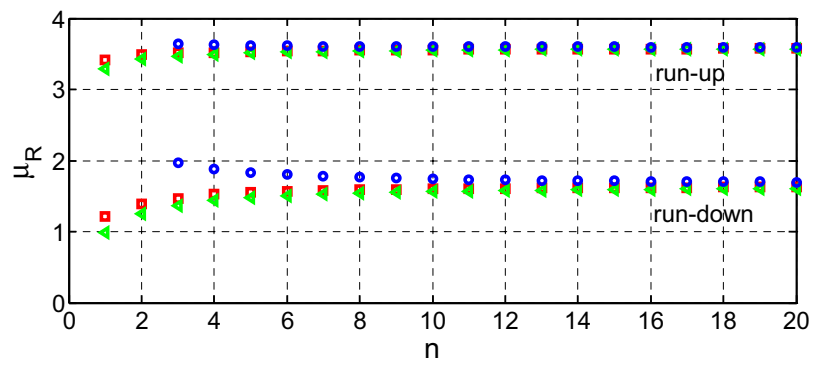

Fig. 3 Wave shape parameter $\mu_{R}$ for different bell-type pulses climbing plane beach: sine-like (blue circles), soliton-like (red squares) and Lorentz-like (green triangles) (color figure online)

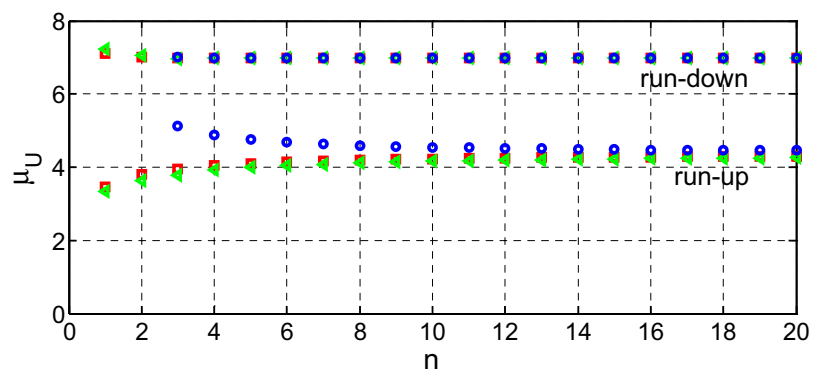

Fig. 4 Wave shape parameter $\mu_{U}$ for different bell-type pulses climbing plane beach: sine-like (blue circles), soliton-like (red squares) and Lorentz-like (green triangles) (color figure online)

$U_{\text {up }}=\mu_{U+} \frac{A L}{\lambda_{s}} \sqrt{\frac{g}{\alpha \lambda_{s}}}, \quad U_{\text {down }}=\mu_{U-} \frac{A L}{\lambda_{s}} \sqrt{\frac{g}{\alpha \lambda_{s}}}$,

where $U_{\text {up }}$ and $U_{\text {down }}$ are maximal run-up and run-down velocities, respectively.

Parameters $\mu_{R}$ and $\mu_{U}$ for bell-shaped waves [Eq. (7)], calculated using the asymptotic analysis $\left(L / \lambda_{s}>>1\right)$ [for details see Didenkulova et al. (2008a)], are demonstrated in Figs. 3 and 4. It can be seen from Figs. 3 and 4 that differences between different pulses for all these parameters are relatively small and can be parameterized. The mean values for shape parameters $\mu_{R}$ and $\mu_{U}$ averaged over the number of pulses together with corresponding standard deviations for different types of pulses and for the entire population are given in Table 1.

Table 1 demonstrates that maximal run-up characteristics show a very weak dependence on the shape of the initial wave and are influenced only by its amplitude $A$ and wavelength $\lambda_{s}$. Thus, expressions for maximal run-up characteristics can be re-written using the mean values of the wave shape parameters:

$$
\begin{aligned}
& R_{\text {up }}=3.6 A \sqrt{\frac{L}{\lambda_{s}}}, \quad R_{\text {down }}=1.6 A \sqrt{\frac{L}{\lambda_{s}}}, \\
& U_{\text {up }}=4.3 \frac{A L}{\lambda_{s}} \sqrt{\frac{g}{\alpha \lambda_{s}}}, \quad U_{\text {down }}=7 \frac{A L}{\lambda_{s}} \sqrt{\frac{g}{\alpha \lambda_{s}}} .
\end{aligned}
$$


Table 1 Calculated shape parameters for different waves climbing plane beach

\begin{tabular}{lllll}
\hline & Sine-like & Soliton-like & Lorentz-like & Total \\
\hline$\mu_{R+}$ & $3.60(1 \pm 0.003)$ & $3.55(1 \pm 0.01)$ & $3.53(1 \pm 0.02)$ & $3.56(1 \pm 0.02)$ \\
$\mu_{R-}$ & $1.76(1 \pm 0.04)$ & $1.56(1 \pm 0.06)$ & $1.51(1 \pm 0.10)$ & $1.60(1 \pm 0.10)$ \\
$\mu_{U+}$ & $4.59(1 \pm 0.04)$ & $4.15(1 \pm 0.05)$ & $4.07(1 \pm 0.06)$ & $4.26(1 \pm 0.07)$ \\
$\mu_{U-}$ & $6.98(1 \pm 0.001)$ & $6.98(1 \pm 0.004)$ & $6.99(1 \pm 0.01)$ & $6.98(1 \pm 0.005)$ \\
\hline
\end{tabular}

Fig. 5 Geometry sketch for wave run-up in a U-shaped bay
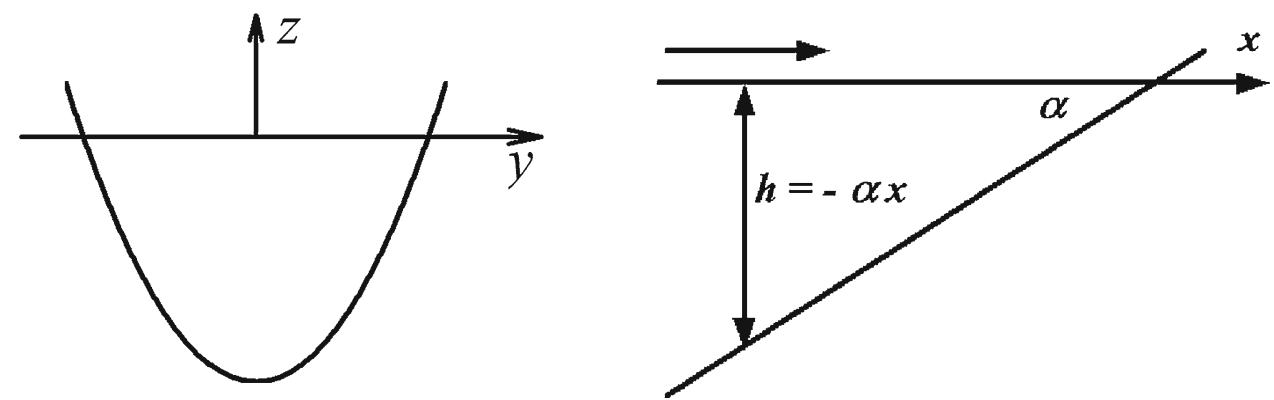

\section{Uncertainty in wave run-up characteristics in a U-shaped bay}

As it has been noted in the Introduction, plane beach model underestimates run-up heights in long and narrow bays (see Didenkulova 2013), and in these cases U-shaped bay model should be applied. Here, we consider a linearly inclined bay of parabolic cross section, see Fig. 5.

The geometry of this bay is described by formula:

$z(x, y)=-\alpha x+\frac{y^{2}}{y_{0}}$,

where $y_{0}$ is an effective width of the channel. Assuming that water flow in the bay is uniform over the cross section, 2D nonlinear shallow water equations for the bay can be reduced to 1D equations (Zahibo et al. 2006; Didenkulova and Pelinovsky 2011a):

$\frac{\partial H}{\partial t}+u \frac{\partial H}{\partial x}+\frac{2 H}{3} \frac{\partial u}{\partial x}=0, \quad \frac{\partial u}{\partial t}+u \frac{\partial u}{\partial x}+g \frac{\partial H}{\partial x}=g \alpha$,

where $H(x, t)=\eta(x, t)-\alpha x$ is a total depth along the main channel axis, $\eta(x, t)$ is water surface displacement, and $u(x, t)$ is water flow averaged over channel cross section.

Equation (12) differs from the classical 1D shallow water equations Eq. (1) only by an additional coefficient $2 / 3$ in the first equation, determined by the parabolic shape of the channel cross section. Similarly to the plane beach, described in Sect. 2, using Riemann invariants and hodograph transformation, Eq. (12) can be reduced to a linear wave equation for the wave function, $\Phi(\lambda, \sigma)$ [similar to Eq. (4)]:

$\frac{\partial^{2} \Phi}{\partial \lambda^{2}}-\frac{\partial^{2} \Phi}{\partial \sigma^{2}}-\frac{2}{\sigma} \frac{\partial \Phi}{\partial \sigma}=0$ where all desired variables are expressed through $\Phi(\lambda, \sigma)$ [similar to Eqs. (2)-(3)]:

$$
\begin{aligned}
& u=\frac{1}{\sigma} \frac{\partial \Phi}{\partial \sigma}, \quad \eta=-\frac{1}{g}\left(\frac{u^{2}}{2}-\frac{1}{3} \frac{\partial \Phi}{\partial \lambda}\right), \\
& x=\frac{1}{g \alpha}\left(\frac{u^{2}}{2}+\frac{\sigma^{2}}{6}-\frac{1}{3} \frac{\partial \Phi}{\partial \lambda}\right), \quad t=\frac{u-\lambda}{g \alpha} .
\end{aligned}
$$

In the U-shaped bay the variable $\sigma$ is also related to the total water depth along the main channel axis $\sigma=\sqrt{6 g H}$ and defines the position of the shoreline $\sigma=0$.

The major difference of the described wave dynamics in U-shaped bay (Zahibo et al. 2006; Didenkulova and Pelinovsky 2011a) from the case of a plane beach (Carrier and Greenspan 1958) is that U-shaped bay allows "nonreflecting" wave propagation, so that waves propagate without inner reflection from the sea bottom and reflect only from the coastline. Mathematically, it corresponds to the travelling wave solution, so that the wave propagates without loss of energy and exerts abnormal wave amplification at the coast (Didenkulova et al. 2008b; Didenkulova and Pelinovsky 2011b). This feature also allows solving run-up problem exactly and finding run-up heights as a function of incident wave shape.

$R_{\text {up }}=2 \tau_{0} \max \left[\frac{\mathrm{d} \eta_{0}}{\mathrm{~d} t}\right]$,

where $\tau_{0}$ is the travel time from a fixed point $x=L$ to the coastline.

As before, we are interested in influence of the wave shape on wave run-up characteristics. For this, we consider again the same types of bell-shaped impulses [see Eq. (7)] propagating in the U-shaped bay. As in Sect. 2, we introduce shape parameters for maximal wave run-up and run-down heights $\mu_{R \pm}$, and run-up and run-down velocities, $\mu_{U \pm}$, based on 
dependences in Eq. (16):

$R_{\text {up } / \text { down }}=\mu_{R \pm} \frac{A L}{\lambda_{s}}, \quad U_{\text {up } / \text { down }}=\mu_{U \pm} \frac{A L \sqrt{g \alpha L}}{\alpha \lambda_{s}^{2}}$,

where $\lambda_{s}$ is the "significant" wavelength in the U-shaped bay.

Calculations of these parameters for different sets of bellshaped pulses Eq. (7) are shown in Figs. 6 and 7. As it was for the plane beach, the error caused by variations in the incident wave shape is minor (see Table 2). The largest variations are observed for a run-up velocity, but even they do not exceed $12 \%$.

Different from the plane beach, maximal run-up and rundown characteristics in the U-shaped bay coincide and lead to a larger oscillations of the shoreline during tsunami event.

Based on calculated shape parameters shown in Table, we can propose generalized formulas for run-up characteristics of solitary pulses in U-shaped bays:

$$
R_{\text {up } / \text { down }}=4.30 \frac{A L}{\lambda_{s}}, \quad U_{\text {up }}=4.56 \frac{A L \sqrt{g \alpha L}}{\alpha \lambda_{s}^{2}},
$$

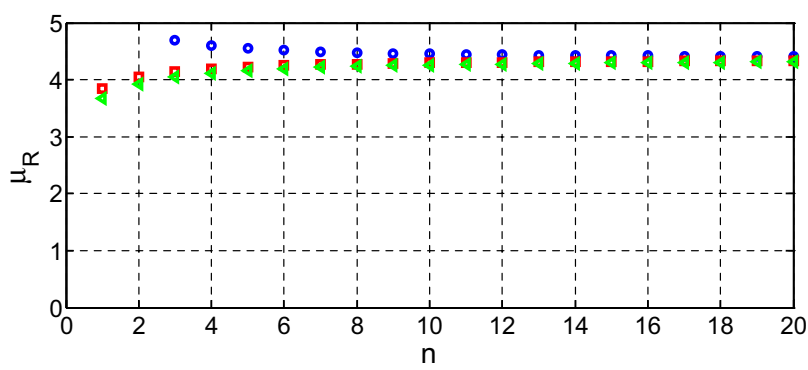

Fig. 6 Wave shape parameter $\mu_{R}$ for different bell-type pulses propagating in U-shaped bay: sine-like (blue circles), soliton-like (red squares) and Lorentz-like (green triangles) (color figure online)

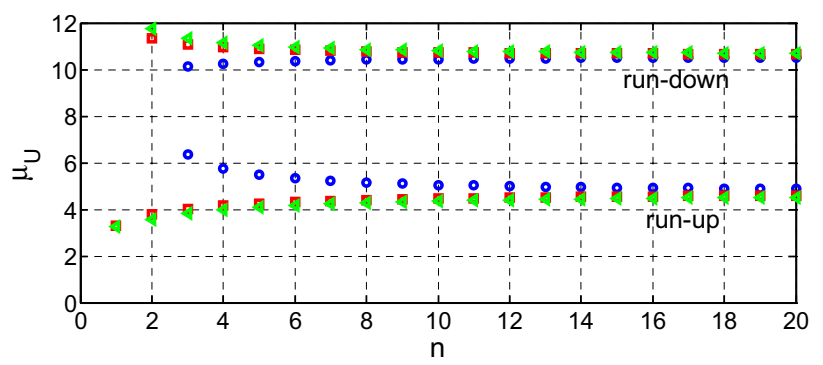

Fig. 7 Wave shape parameter $\mu_{U}$ for different bell-type pulses propagating in U-shaped bay: sine-like (blue circles), soliton-like (red squares) and Lorentz-like (green triangles) (color figure online)

$$
U_{\text {down }}=10.78 \frac{A L \sqrt{g \alpha L}}{\alpha \lambda_{s}^{2}}
$$

It should also be noted that formulas for a plane beach Eq. (10) and for U-shaped bay Eq. (18) can also be used for pulses of negative polarity. In this case, the pares $R_{\text {up }}$ and $R_{\text {down }}$ and $U_{\text {up }}$ and $U_{\text {down }}$ should be swapped.

\section{Wave asymmetry and steepness of the wave front}

Although small variations in the incoming wave of symmetric bell-like shape do not lead to significant changes in its run-up characteristics, the influence of wave asymmetry can be significant. It has been theoretically shown (Didenkulova et al. 2007b) and confirmed experimentally in Didenkulova et al. (2013) that increase in wave front steepness, defined as maximum time derivative of the wave shape $\eta[\max (\partial \eta / \partial t)]$, can increase maximal run-up height substantially (see Fig. 8). For a sine wave, this influence follows the square rooting law for maximal wave run-up:

$R_{\text {up }} \sim \sqrt{s}$

where $s$ is the front steepness of the deformed wave at the beginning of the slope and $s_{0}$ is the front steepness of the initial (not deformed) wave.

This theoretical dependence is also supported by experimental data Didenkulova et al. (2013). The experiment was conducted in the $300 \mathrm{~m}$ long Large Wave Flume (GWK, FZK) in Hannover, Germany. Sinusoidal waves were propagating $251 \mathrm{~m}$ in the basin of constant depth of $3.5 \mathrm{~m}$ and then were climbing 1:6 slope. Figure 8 demonstrates that experimental points nicely follow the theoretical curve.

Similar effects can also be observed in the U-shaped bay. Due to general wave intensification in U-shaped bays, the influence of wave front steepness on wave run-up characteristics is also stronger. According to Didenkulova and Pelinovsky (2011a), maximal run-up height has a linear dependence on wave front steepness, so that formula (18) can be re-written taking into account the effect of wave asymmetry:

$R_{\text {up }}=4.30 \frac{A L}{\lambda_{s}} \frac{s}{s_{0}}$.
Table 2 Calculated shape parameters for different waves propagating in U-shaped bay

\begin{tabular}{lrrrr}
\hline & \multicolumn{1}{l}{ Sin-like } & \multicolumn{1}{l}{ Soliton-like } & \multicolumn{1}{l}{ Lorentz-like } & \multicolumn{1}{c}{ Total } \\
\hline$\mu_{R \pm}$ & $4.47(1 \pm 0.02)$ & $4.25(1 \pm 0.03)$ & $4.20(1 \pm 0.04)$ & $4.30(1 \pm 0.04)$ \\
$\mu_{U+}$ & $5.16(1 \pm 0.07)$ & $4.35(1 \pm 0.08)$ & $4.24(1 \pm 0.08)$ & $4.56(1 \pm 0.12)$ \\
$\mu_{U-}$ & $10.43(1 \pm 0.01)$ & $10.86(1 \pm 0.03)$ & $11.01(1 \pm 0.05)$ & $10.78(1 \pm 0.04)$ \\
\hline
\end{tabular}




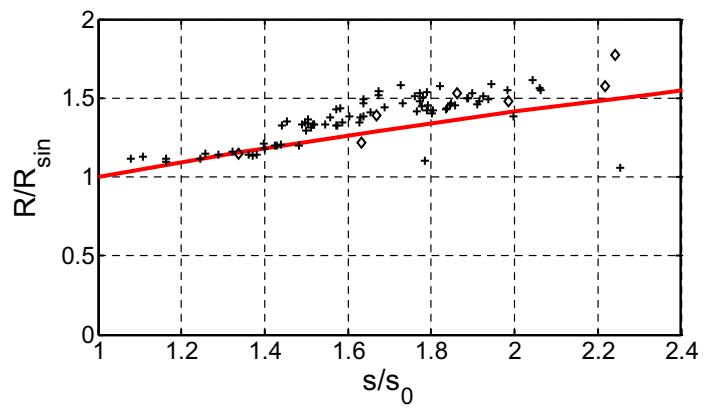

Fig. 8 Influence of wave front steepness on its maximal run-up and run-down heights: solid line corresponds to the theoretical dependence and crosses - to the experimental measurements

\section{Conclusion}

In this paper, we discuss how changes in the shape of incoming tsunami wave influence its run-up in different bay geometries: plane beach and U-shaped bay.

It has been demonstrated that for symmetric solitary pulses formulas for run-up characteristics in both plane beach and U-shaped bay are rather stable and can be generalized for all positive or negative pulses. The corresponding formulas are Eqs. (10) and (18). At the same time, asymmetry of the wave and in particular the steepness of the wave front play crucial role and increases run-up height substantially. In a plane beach geometry tsunami run-up height is proportional to the square root of the wave front steepness. In a U-shaped bay which allows nonreflecting wave propagation (Didenkulova and Pelinovsky 2009, 2011a), all processes are intensified and influence of the wave front steepness on run-up height is also stronger and is represented by linear dependence.

It should be noted that parameterization presented in this paper works only for symmetric waves, such as bell-shaped impulses of positive or negative polarity. For asymmetric impulses, such as $N$-waves or nonlinear deformed waves, additional parameters characterizing the wave shape should be taken into account. As it is shown in this manuscript, for nonlinear deformed waves such parameter is the wave front steepness. More complicated wave shapes may also depend on other additional parameters.

Acknowledgments The results presented in this paper are obtained with the support of State Contract No. 2014/133 and Grants RFBR (14-02-00983, 14-05-00092), MK-1146.2014.5 and SF0140007s11. Authors also acknowledge the support from CENS through the European Regional Development Fund (ERDF). Some aspects of the appearance of the extreme run-up characteristics are considered in the framework of a Volkswagen grant.

\section{References}

Antuono M, Brocchini M (2007) The boundary value problem for the nonlinear shallow water equation. Stud Appl Math 119:71-91
Antuono M, Brocchini M (2008) Maximum run-up, breaking conditions and dynamical forces in the swash zone: a boundary value approach. Coast Eng 55:732-774

Antuono M, Brocchini M (2010) Solving the nonlinear shallow-water equations in physical space. J Fluid Mech 643:207-232

Aranguiz R, Shibayama T (2013) Effect of submarine canyons on tsunami propagation: a case study of the Biobio canyon, Chile. Coast Eng J 55:1350016

Brocchini M, Gentile R (2001) Modelling the run-up of significant wave groups. Cont Shelf Res 21:1533-1550

Carrier G, Greenspan H (1958) Water waves of finite amplitude on a sloping beach. J Fluid Mech 4:97-109

Carrier G, Wu T, Yeh H (2003) Tsunami run-up and draw-down on a plane beach. J Fluid Mech 475:79-99

Didenkulova I (2009) New trends in the analytical theory of long sea wave runup. In: Quak E, Soomere T (eds) Applied wave mathematics: selected topics in solids, fluids, and mathematical methods. Springer, Berlin, pp 265-296

Didenkulova I (2013) Tsunami runup in narrow bays: the case of Samoa 2009 tsunami. Nat Hazards 65:1629-1636

Didenkulova I, Pelinovsky E (2009) Non-dispersive traveling waves in strongly inhomogeneous water channels. Phys Lett A 373:38833887

Didenkulova I, Pelinovsky E (2011a) Nonlinear wave evolution and runup in an inclined channel of a parabolic cross-section. Phys Fluids 23:086602

Didenkulova I, Pelinovsky E (2011b) Runup of tsunami waves in Ushaped bays. Pure Appl Geophys 168:1239-1249

Didenkulova I, Kurkin A, Pelinovsky E (2007a) Run-up of solitary waves on slopes with different profiles. Izv Atmos Ocean Phys 43:384-390

Didenkulova I, Pelinovsky E, Soomere T, Zahibo N (2007b) Runup of nonlinear asymmetric waves on a plane beach. In: Kundu A (ed) Tsunami and nonlinear waves. Springer, Berlin, pp 175190

Didenkulova I, Pelinovsky E, Soomere T (2008a) Run-up characteristics of tsunami waves of "unknown" shapes. Pure Appl Geophys 165(11/12):2249-2264

Didenkulova I, Pelinovsky E, Zahibo N (2008b) Reflection of long waves from a "non-reflecting" bottom profile. Fluid Dyn 43:101107

Didenkulova I, Denissenko P, Rodin A, Pelinovsky E (2013) Effect of asymmetry of incident wave on the maximum runup height. $\mathrm{J}$ Coast Res 65:207-212

Kânoğlu U (2004) Nonlinear evolution and runup-drawdown of long waves over a sloping beach. J Fluid Mech 513:363-372

Kânoğlu U, Synolakis C (2006) Initial value problem solution of nonlinear shallow water-wave equations. Phys Rev Lett 97:148501

Lauterjung J, Münch U, Rudloff A (2010) The challenge of installing a tsunami early warning system in the vicinity of the Sunda Arc, Indonesia. Nat Hazards Earth Syst Sci 10:641-646

Løvholt F, Glimsdal S, Harbitz C B, Zamora N, Nadim F, Peduzzi P, Dao H I, Smebye H (2012) Tsunami hazard and exposure on the global scale. Earth Sci Rev 110:58-73

Madsen P, Fuhrman D (2008) Run-up of tsunamis and periodic long waves in terms of surf-similarity. Coast Eng 55:209223

Okal E, Fritz H, Synolakis C, Borrero J, Weiss R, Lynett P, Titov V, Foteinis S, Jaffe B, Liu PL-F, Chan I-Ch (2010) Field survey of the Samoa tsunami of 29 September 2009. Seismol Res Lett 81:577591

Pedersen G, Gjevik B (1983) Runup of solitary waves. J Fluid Mech 142:283-299

Pedersen GK, Lindstrom E, Bertelsen AF, Jensen A, Laskovski D, Saelevik G (2013) Runup and boundary layers on sloping beaches. Phys Fluids 25:012102 
Pelinovsky E, Mazova R (1992) Exact analytical solutions of nonlinear problems of tsunami wave run-up on slopes with different profiles. Nat Hazards 6:227-249

Rybkin A, Pelinovsky E, Didenkulova I (2014) Nonlinear wave run-up in bays of arbitrary cross-section: generalization of the CarrierGreenspan approach. J Fluid Mech 748:416-432

Spielvogel L (1975) Runup of single waves on a sloping beach. J Fluid Mech 74:685-694

Synolakis C (1987) The runup of solitary waves. J Fluid Mech 185:523545
Tadepalli S, Synolakis C (1994) The runup of N-waves. Proc R Soc Lond A445:99-112

Tinti S, Tonini R (2005) Analytical evolution of tsunamis induced by near-shore earthquakes on a constant-slope ocean. J Fluid Mech 535:33-64

Vasskog K, Waldmann N, Bondevik S et al (2013) Evidence for Storegga tsunami run-up at the head of Nordfjord, Western Norway. J Q Sci 28:391-402

Zahibo N, Pelinovsky E, Golinko V, Osipenko N (2006) Tsunami wave runup on coasts of narrow bays. Int J Fluid Mech Res 33:106-118 